\title{
Stochastic Channel Selection in Cognitive Radio Networks
}

\author{
Yang Song and Yuguang Fang \\ Department of Electrical and Computer Engineering \\ University of Florida \\ Gainesville, Florida 32611 \\ Email: \{yangsong@,fang@ece.\}ufl.edu
}

\author{
Yanchao Zhang \\ Department of Electrical and Computer Engineering \\ New Jersey Institute of Technology \\ University Heights, Newark, NJ 07102 \\ Email: yczhang@njit.edu
}

\begin{abstract}
In this paper, we investigate the channel selection strategy for secondary users in cognitive radio networks. We claim that in order to avoid the costly channel switchings, a secondary user may desire an optimal channel which maximizes the probability of successful transmissions, rather than consistently adapting channels to the random environment. We propose a stochastic channel selection algorithm based on the learning automata techniques. This algorithm adjusts the probability of selecting each available channel and converges to the $\epsilon$-optimal solution asymptotically.
\end{abstract}

\section{INTRODUCTION}

In wireless communication, the most valuable resource is the available radio frequencies. The dramatic increase in wireless services aggravates the scarcity of the frequency spectrum. In Unite States, the Federal Communications Commission (FCC) regulates the frequency spectrum and allocates it in an exclusive-usage fashion. Currently, the spectrum is overcrowded and there is few space available for the new emerging wireless services. Ironically, as reported in a set of real experiments [1], the majority of the current allocated spectrum is severely under-utilized. For example, in [1], it is observed that, on average, there is only about $5.2 \%$ of the spectrum below $3 \mathrm{GHz}$ is actually in use. The ubiquitous "spectrum holes" and the growth of new wireless services motivate the DAPRA to start the XG program [2] in order to investigate the policies and regulation rules which enable the spectrum holes to be utilized by other users, a.k.a., secondary user, more efficiently. The new scheme is termed as opportunistic spectrum access (OSA) or dynamic spectrum access (DSA) in general.

In order to dynamically sense the frequency spectrum and adjust the operating frequency, the cognitive radios are proposed as the solution to fully utilize the valuable spectrum. Cognitive radios, usually based on the software defined radio (SDR) techniques, are able to detect the current spectrum, sense the spectrum holes and adjust the parameters, such as frequency, power and transmission rate, in order to use the spectrum more efficiently and economically. For example, a novel IEEE 802.22 standard is introduced in [3], which enables the cognitive radios to utilize the unused analog TV broadcast

This work was supported in part by the U.S. National Science Foundation under Grant DBI-0529012 and under Grant CNS-0626881. band as secondary users. They are allowed to operate at the TV broadcast band whenever the primary user, i.e., TV broadcast signal, is absent. Moreover, if there are multiple primary users exist, i.e., there are multiple frequency opportunities available, the cognitive radios can dynamically switch among all available frequencies following the unpredictable returns of the primary users. There are several MAC protocols proposed in the literature [4] for cognitive radio networks, which serve as the guidelines of switching channels according the environmental changes. However, few of them take account of the drastic cost of changing frequencies in current manufactured wireless devices. The aggravated delay and the deteriorated packet-lossratio induced by the channel switchings have caught more and more attention in the community [5] [6] [7] [8] [9] [10] [11]. For example, a recent experimental study [12] shows that, on average, channel switchings can cause up to a packet loss ratio of $3 \%$, exclusively. In cognitive radio networks, the situation is even worse. Due to the random characteristics of primary users, the secondary user with frequency-agile MAC protocols needs to switch the operating frequency adaptively and consistently. Therefore, the long-term performance, in terms of QoS support and aggregated throughput, will be dramatically degraded due to the overwhelming cost in channel switchings. Thus, from the secondary user's point of view, it may be more favorable to implement a channel selection strategy which reluctates to switch the channels, unless necessary. Moreover, note that the essential rationale of adapting channels is to minimize the conflicts with the primary users. Hence, we claim that the prudent strategy for a secondary user is to insist on the statistically optimal channel which maximizes the probability of successful transmissions, rather than adaptively switching channels as the random and unpredictable behaviors of primary users fluctuate.

However, the problem of acquiring the optimal channel is by no means straightforward. Were the distributions of returning time and operating duration of primary users known as a prior, the problem is easy to solve, yet this knowledge is not attainable in practical cognitive radio networks. In this paper, we propose a novel stochastic channel selection algorithm based on learning automata (LA), which dynamically adjusts the probability of choosing one channel on the fly and asymptotically converges to the optimal channel, in the sense 
that the probability of successful transmissions is maximized.

The rest of this paper is organized as follows. Section II briefly overviews the background and the motivation of this paper. The stochastic channel selection algorithm is proposed in Section III. An illustrative example of the algorithm is provided in Section IV. Finally, Section V concludes this paper.

\section{BACKGROUND AND MOTIVATION}

\section{A. Cognitive Radio Networks}

In the literature, the dynamic spectrum access schemes are categorized into three classes [13]: exclusive use model, open sharing model and hierarchical access model. The exclusive use model represents the scenarios where the primary users can sublease part of the private spectrum to other secondary users for profit. Each user, either primary or secondary, does not share the spectrum with other users. However, in the open sharing model, all the users obey a certain spectrum etiquette and share the spectrum with each other. The unlicensed ISM band is a well-known example. In the hierarchical access model, primary users and secondary users coexist and the secondary users can transmit only when the summed interference measured at the primary user's receiver is below a certain threshold. For detailed overview of the dynamic spectrum access schemes for cognitive radio networks, one may refer to [14] and a new comprehensive survey in [13].

In our work, we consider a hierarchical access model of cognitive radio networks with minimum interference tolerance where the secondary users are required to terminate the current transmission immediately after the corresponding primary user returns. In the network, there are multiple primary users with different frequencies and each secondary user is a pair of transmitter and receiver which are both cognitive radios. We assume that the secondary users have the capability of sensing the spectrum and the willingness of terminating the current transmission whenever the primary user returns. The traffic generated on each secondary user is transmitted over consequent time slots. At the beginning of each time slot, the loaded secondary user selects one of the available frequencies as the transmission frequency, and transmits. If the corresponding primary user, i.e., the one who owns the selected channel, does not return within this time slot, the transmission is successful. On the contrary, if the primary user returns, the current transmission will be terminated and the secondary user needs to retransmit all the packets in the next available time slot. We also consider the influence of other secondary users on the channel selection strategy of this particular secondary user. Apparently, whether the transmission of this particular secondary user, say $k$, is successful or not depends not only the primary users but also the neighboring users of $k$ as well. The definition of neighboring users depends on the specific spectrum etiquette. For example, if we use the traditional carrier sensing mechanism, the neighboring users of $k$ is defined as the secondary users (1) whose transmitter is within the carrier sensing range of the $k$-th receiver, or (2) whose receiver is within the carrier sensing range of the $k$-th transmitter. Thus, we define that for the $k$-th secondary user, in a time slot, the transmission is successful if (1) at the beginning of the slot, there is no neighboring users ${ }^{1}$ operating at the same frequency channel as $k$ has selected, and (2) the primary user who owns this channel does not return within the current time slot, and define as failure otherwise.

From the $k$-th secondary user's perspective, the strategy of dynamically adapting channels according to the random environment, which consists of all primary users and coexisting secondary users, is likely unfavorable, owing to the dominant and burdensome cost in channel switchings. Instead, one prudent secondary user may want to find the optimal channel in terms of maximizing the probability of successful transmissions. Hence, the expected throughput is maximized by insisting on the statistically optimal channel, if the transmission rate is assumed fixed. Besides, there is no more costly channel switchings once the optimal channel is obtained. However, as mentioned above, the problem of acquiring the optimal channel from a zero-knowledge on the statistics of the random environment, which consists of nondeterministic primary users and unpredictable coexisting secondary users, is challenging. Fortunately, the learning automata (LA) provides us a family of algorithmic tools which converge to the optimum strategy asymptotically in arbitrary stationary random environments with no prior distribution knowledge required. We will briefly overview the principles of the learning automata in the next subsection.

\section{B. Learning Automata}

In classical control theory, the mathematical model for a system is usually assumed to be known. The expected output of the current input can be predicted in a deterministic fashion. Although the later developed stochastic control theory deals with the issues of uncertain parameters, they both assume an unchanging system. Stochastic learning approaches are introduced to solve the problems of random systems. The scenarios where learning automata techniques can be applied are summarized as follows [15]. In a random environment, there are finite actions available for the decision maker to choose from. Each deterministic action induces a random output from the random environment, which could be either favorable or unfavorable. Based on the observations, an LA algorithm is expected to determine a strategy of selecting actions at a stage given the past actions and corresponding outputs. We can view the random environment as a black box, depicted in Figure 1. At each decision instance, the decision maker picks an action according a certain rule as the input to the random environment and waits for the random output. Apparently, if we are patient enough, we could execute voluminous trials for each action, say a million times, to estimate the optimal action in terms of maximum probability of getting rewards. The beauty of the LA approaches lies in that they can converge to the $\epsilon$-optimal solution with a reasonable fast pace for

\footnotetext{
${ }^{1}$ In general, the neighboring users of $k$ is defined as the secondary users whose actions may potentially cause a transmission failure of user $k$.
} 


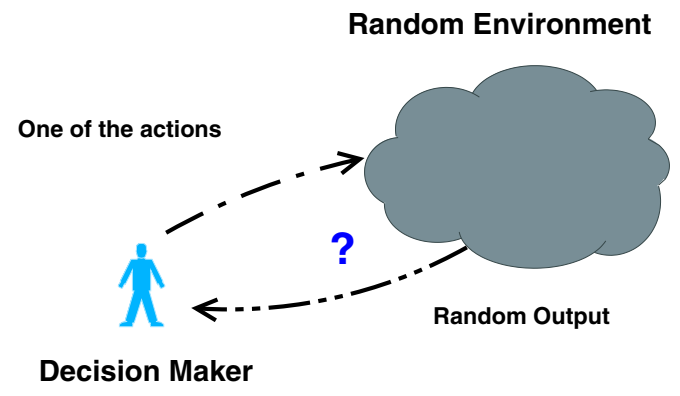

Fig. 1. The interaction between the decision maker and the random environment.

arbitrary stationary random environments. In addition, the LA approaches can also track the optimal action for non-stationary environments as long as the statistic characteristics of the environment does not change very fast. Recently, the learning automata techniques have been extensively applied in various aspects in the communication and networking communities [16] [17] [18] [19] [20]. For an overview of learning automata techniques, refer to [21].

Let us revisit the paradigm of cognitive radio networks. From the $k$-th secondary user's perspective, the "outer world" can be viewed as a random environment. The randomness in the response from this outer world is induced by both the unpredictable returns of primary users and the uncontrollable behaviors of neighboring users. We consider this complex outer world for the secondary user $k$ as a "black box". At the beginning of each transmission slot, the secondary user selects one of the available channels. The output of this black box is a binary number $\beta$ where $\beta=0$ denotes the transmission is successful and $\beta=1$ otherwise. In the next section, we will propose an LA-based stochastic channel selection algorithm for secondary users in cognitive radio networks. The convergence property of the algorithm will also be discussed.

\section{Stochastic Channel Selection}

We consider a stationary ${ }^{2}$ cognitive radio network where $M$ primary users and $C$ secondary users coexist, illustrated in Figure 2. Each secondary user is a cognitive transmitter-

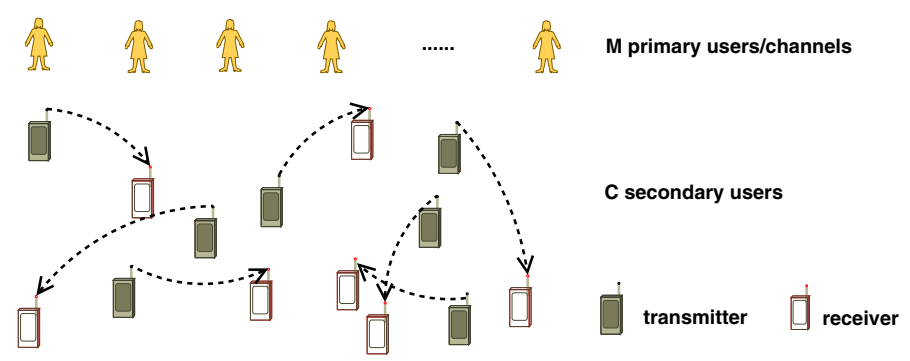

Fig. 2. Topology of the cognitive radio network.

\footnotetext{
${ }^{2} \mathrm{We}$ assume the random environment is stationary, however, the proposed stochastic channel selection algorithm can track the optimal solution in slow statistic-varying environment as well.
}

receiver pair which can adjust the transmission frequency among $1, \cdots, M$ indexed channels. The transmission of the primary users are protected by enforcing the secondary users to exit immediately whenever the legitimate primary user comes back. For simplicity, we assume that each secondary user has a fixed transmission rate. The traffic generated at the transmitter of the $k$-th secondary user is transmitted to the receiver in consequent time slots. We denote the starting time of each slot as $T_{i}$ where $i$ is the slot number. Each secondary user, say $k$, keeps a probability vector $\mathbf{P}_{k}=\left[p_{1}, \cdots, p_{M}\right]$ and an estimation vector $\mathbf{D}_{k}=\left[d_{1}, \cdots, d_{M}\right]$, where $p_{i}$ is the probability of choosing channel $i$ and $d_{i}$ is the estimated possibility of successful transmission by selecting channel $i$. At the beginning of a slot, say $m$-th slot, the transmitter picks a channel according to the probability vector $\mathbf{P}_{k}$. If the selected channel is busy, i.e., the primary user is present, the same process repeats until an available channel is selected and transmission begins. If the transmission is successful, user $k$ will set $\beta=0$ and $\beta=1$ otherwise. Based on the received value of $\beta$, the user $k$ updates the probability vector $\mathbf{P}_{k}$ and the estimation vector $\mathbf{D}_{k}$. Then enters the next time slot $T_{m+1}$.

The algorithm executed in the $k$-th secondary users is provided in detail as follows.

\section{Parameters: $^{3}$}

- $R$ : The resolution parameter (tunable).

- $W$ : The initialization parameter (tunable).

- $M$ : The number of primary users, i.e., the number of channel opportunities.

- $C$ : The number of secondary users.

- $H(n)$ : The number of channels which have higher values in the estimation vector $D$ than the current selected channel.

- $\delta$ : The step size of adjusting the probability vector and $\delta=\frac{1}{R}$.

- $S_{i}(n)$ : The number of slots where the transmissions with channel $i$ are successful, up to $T_{n}$.

- $C_{i}(n)$ : The number of slots where the channel $i$ are chosen to transmit packets, up to $T_{n}$.

\section{Algorithm:}

\section{Initialization:}

- The user $k$ sets $P(0)=\left[p_{1}, \cdots, p_{M}\right]$ where $p_{i}=\frac{1}{M}$ for all $1 \leq i \leq M$.

- Chooses an available channel according to $P(0)$ then transmits and records the values of $\beta \mathrm{s}$, until each channel is selected $W$ times.

- Depending on the values of $\beta$ s, i.e., whether the corresponding transmissions were successful, sets $S_{i}(0)$ and $C_{i}(0)$ for each channel $i$.

- Initializes $D(0)=\left[d_{1}, \cdots, d_{M}\right]$ where $d_{i}=\frac{S_{i}(0)}{C_{i}(0)}$.

\section{Do:}

${ }^{3}$ We omit the subscript $k$ in the following algorithm description. 
- Selects an available transmission channel $i$ according to the probability vector $P(n)$.

- Update the probability vector $P(n)$ according to the following equations.

$$
\begin{aligned}
p_{j}(n+1)= & \min \left(p_{j}(n)+\frac{\delta}{H(n)}, 1\right) \\
& \forall j \text { if } d_{j}(n)>d_{i}(n) . \\
p_{j}(n+1)= & \max \left(p_{j}(n)-\frac{\delta}{M-H(n)}, 0\right) \\
& \forall j \text { if } d_{j}(n)<d_{i}(n) \\
p_{i}(n+1)= & 1-\sum_{j \neq i} p_{j}(n+1)
\end{aligned}
$$

- After $T_{n}$, the secondary user adjusts the running estimation of the successful transmission possibility, i.e., $D$, by

$$
\begin{aligned}
S_{i}(n+1) & =S_{i}(n)+(1-\beta) \\
C_{i}(n+1) & =C_{i}(n)+1 \\
d_{i}(n+1) & =\frac{S_{i}(n+1)}{C_{i}(n+1)}
\end{aligned}
$$

where $\beta=0$ if the transmission is successful and $\beta=1$ otherwise.

\section{Until:}

- $\max (P(n))>B$ where $B$ is a predefined convergence threshold.

\section{End.}

The channel selection algorithm introduced above falls into the family of the Discrete Generalized Pursuit Algorithms (DGPA) defined in [22] where the convergence speeds of various learning automata approaches are compared numerically. It is shown that DGPA converges remarkably faster than other LA-based algorithms. Moreover, the $\epsilon$-optimality of DGPA is proved in [22], based on the earlier results in [23]. Thus, we provide the following theorem for the stochastic channel selection algorithm without proof due to the page limit.

Theorem 1: The stochastic channel selection algorithm is $\epsilon$-optimal for any stationary cognitive radio networks. In other words, for any arbitrarily small $\epsilon>0$ and $\gamma>0$, there exists a $n^{\prime}$ satisfying

$$
\operatorname{Pr}\left\{\left|1-p_{z}(n)\right|<\epsilon\right\}>1-\gamma \forall n>n^{\prime}
$$

where $z$ is the index of the optimal channel in terms of probability of successful transmissions.

\section{An Illustrative Example}

In this section, we use a simple example to illustrate the effectiveness of the stochastic channel selection algorithm introduced in the previous section.

For simplicity, we consider a single-secondary-user cognitive radio network with consistent traffic load. In other words, the uncertainty of the random environment comes solely from the unpredictable returns of the primary users. We assume that there are five primary users with distinct channels. The traffic on the secondary user is transmitted over successive time slots. The returning probability vector $\varphi$ consists of five probabilities, i.e., $\varphi=\left[p_{1}, \cdots, p_{5}\right]$ where $p_{i}$ denotes the probability of returning of the $i$-th primary user in each slot. Without loss of generality, we assume that the primary users have short jobs and hence the durations are within one time slot. The simulation parameters are summarized as follows.

- $\mathrm{R}$ : The resolution parameter is set to $R=50$.

- W: The initialization parameter is set to $W=10$.

- M: There are five primary users, i.e., $M=5$.

- C: There is a single secondary user, i.e., $C=1$.

- $\delta$ : The step size is set to $\delta=\frac{1}{R}=0.02$.

- B: The convergence threshold is set to $B=0.9999$.

We assume that the returning probability vector is $\varphi=$ $[0.2,0.1,0.3,0.4,0.3]$. By inspection, we observe that the second primary user has the least possibility of returning in each slot. Therefore, channel 2 is the optimal strategy in the sense that the probability of successful transmissions is maximized. The stochastic channel selection algorithm implemented in the secondary user is expected to find this optimal strategy asymptotically, which is verified in Figure 3.

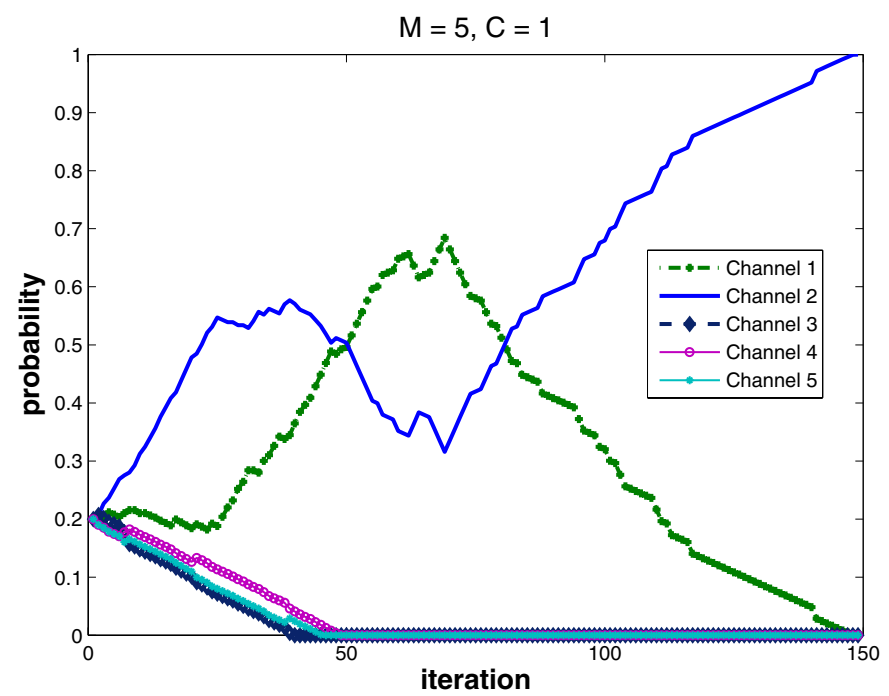

Fig. 3. The probability updating history of the stochastic channel selection algorithm.

Figure 3 depicts the trajectories of the updating processes of channel selection probabilities. At the initialization step, each channel is assigned with an equal probability. We notice that channel $3,4,5$ are eliminated by the stochastic channel selection algorithm soon. Channel 1 has been preserved by the algorithm since $p_{1}=0.2$ and it is the second-best candidate to the optimal strategy, i.e., channel 2 where $p_{2}=0.1$. Interestingly, in a certain period of time, channel 1 outperforms channel 2 dramatically, e.g., at around the 70-th iteration, the probabilities of selecting channel 1 and 2 are 0.7 v.s. 
0.3, as denoted in Figure 3. However, the stochastic channel selection algorithm leans to the optimal strategy gradually and the probability of selecting the optimal channel approaches 1 asymptotically, as expected.

\section{CONCLUSIONS}

We consider the design issues of optimal channel selection for secondary users in cognitive radio networks. We claim that the overwhelming cost in frequent channel-switchings, in terms of aggravated delay and packet loss ratio, makes the schemes with less consistent switchings more appealing and favorable. We propose a stochastic channel selection algorithm based on the learning automata techniques. This algorithm asymptotically converges to the $\epsilon$-optimal strategy, in the sense that the probability of successful transmissions is maximized. Our analysis shows the effectiveness of the proposed algorithm.

\section{REFERENCES}

[1] [Online]. Available: http://www.sharedspectrum.com

[2] [Online]. Available: http://web-ext2.darpa.mil/sto/smallunitops/xg.html

[3] C. Cordeiro, K. Challapali, D. Birru, and S. S. N, "Ieee 802.22: the first worldwide wireless standard based on cognitive radios," First IEEE International Symposium on New Frontiers in Dynamic Spectrum Access Networks, DySPAN05', pp. 328- 337, Nov. 2005.

[4] Proceedings of the first IEEE Symposium on New Frontiers in Dynamic Spectrum Access Networks, Nov.2005.

[5] P. Kyasanur, J. So, C. Chereddi, and N. H. Vaidya, "Multi-channel mesh networks: Challenges and protocols," IEEE Wireless Communications, Apr.2006, invited paper.

[6] J. Shi, T. Salonidis, and E. W.Knightly, "Starvation mitigation through multi-channel coordination in csma multi-hop wireless networks," Proceedings of the seventh ACM international symposium on Mobile ad hoc networking and computing, MobiHoc'06, pp. 214-225, 2006.

[7] P. Kyasanur and N. H. Vaidya, "Capacity of multichannel wireless networks: Impact of channels, interfaces, and interface switching delay," University of Illinois at Urbana-Champaign, Tech. Rep., Oct.2006.

[8] V. Bhandari and N. H.Vaidya, "Connectivity and capacity of multichannel wireless networks with channel switching constraints," Proceedings of IEEE INFOCOM'07.

[9] J. A. Patel, H. Luo, and I. Gupta, "A cross-layer architecture to exploit multi-channel diversity with a single transceiver," Proceedings of IEEE INFOCOM'07.

[10] P. Kyasanur and N. H.Vaidya, "Routing in multi-channel multi-interface ad hoc wireless networks," University of Illinois at Urbana-Champaign, Tech. Rep., 2004.

[11] C. Chereddi, "System architecture for multichannel multi-interface wireless networks," Master's thesis, University of Illinois at UrbanaChampaign, 2006.

[12] P. Li, N. Scalabrino, and Y. Fang, "Channel switching cost in multichannel wireless mesh networks," 2007, to appear in Milcom'07.

[13] Q. Zhao and B. M. Sadler, "A survey of dynamic spectrum access: Signal processing, networking, and regulatory policy," IEEE Signal Processing Magazine, May 2007.

[14] I. F. Akyildiz, L. W.Y., V. M.C., and M. S., "Next generation/dynamic spectrum access/cognitive radio wireless networks: A survey," Computer Networks Journal, (Elsevier), Sep. 2006.

[15] C. Unsal, "Intelligent navigation of autonomous vehicles in an automated highway system: Learning methods and interacting vehicles approach," Ph.D. dissertation, Virginia Polytechnic Institute and State University, 1998, chapter 3 : Stochastic Learning Automata.

[16] Y. Xing and R. Chandramouli, "Price dynamics in competitive agile spectrum access markets," IEEE Journal on Selected Areas in Communications, Mar.2007.

[17] M. A. Haleem and R. Chandramouli, "Adaptive downlink scheduling and rate selection: A cross layer design," IEEE Journal on Selected Areas in Communications, vol. 23, Jun. 2005.
[18] _ - "Adaptive transmission rate assignment for fading wireless channels with pursuit learning algorithm," Proceedings of CISS, Princeton, 2004.

[19] S. Kiran and R. Chandramouli, "An adaptive energy-efficient link layer protocol using stochastic learning control," IEEE International Conference on Communications (ICC), 2003.

[20] Y. Xing and R.Chandramouli, "Distributed discrete power control for bursty transmissions over wireless data networks," IEEE International Conference on Communications (ICC), 2004.

[21] M. A. L. Thathachar and P. S. Sastry, "Varieties of learning automata: An overview," IEEE Transactions on systems, man, and cybernetics, vol. 32, Dec.2002.

[22] M. Agache and B. Oommen, "Generalized pursuit learning schemes: New families of continuous and discretized learning automata," IEEE Transactions on systems, man, and cybernetics, vol. 32, Dec.2002.

[23] B. J. Oommen and J. K. Lanctot, "Discretized pursuit learning automata," IEEE Transactions on systems, man, and cybernetics, vol. 20, Aug. 1990 